\title{
Computationally Efficient Millimeter-Wave Backscattering Models: A Single-Scattering Model
}

\author{
Adrián Lahuerta-Lavieja, Student Member, IEEE, Martin Johansson, Senior Member, IEEE, Ulf Gustavsson, \\ Thomas A. H. Bressner, and Guy A. E. Vandenbosch, Fellow, IEEE
}

\begin{abstract}
The use of millimeter-wave (mm-wave) frequency bands for fifth-generation cellular mobile communications (5G) has led to intense interest from academia and industry over these spectrum resources. Despite extensive measurement campaigns and channel modeling efforts, there is a lack of deterministic backscattering models addressing the impact of the size and orientation of static scatterers on the radio channel. In this paper, two three-dimensional (3D) computationally-efficient methods for calculating backscattering based on the Fresnel integrals and the error function are proposed and validated both against simulations and measurements. In addition, applying the same methodology, state-of-the-art mm-wave blockage (forwardscattering) models are modified to capture backscattered fields. Furthermore, both the introduced and the modified models preserve the structure of geometry-based stochastic channel models (GSCMs) and thus their implementation in system-level simulators is substantially beneficial due to their good accuracy and short computation time.
\end{abstract}

Index Terms-5G mobile communication, backscattering, channel model, computational complexity, diffraction, error function, Fresnel integral, millimeter wave propagation

\section{INTRODUCTION}

$\mathbf{F}$ IFTH-GENERATION cellular mobile communications (5G), with 1000 times aggregate-data-rate enhancement with respect to current cellular systems, below-1-ms latency, and the possibility of an internet of things (IoT) comprised of billions of miscellaneous devices, is foreseen to drastically transform the communications ecosystem [1]. This is pushing industry and academia to explore new solutions and technologies to make the new generation of wireless communications a reality.

Due to the high bandwidth availability, the millimeterwave (mm-wave) frequency band has been proposed as a solution to achieve the required data rates [2]. Therefore, the

Manuscript received June 7, 2019; revised September 22, 2019; accepted December 25, 2019. Date of publication April 14, 2020; date of current version August 4, 2020. This project has received funding from the European Union's Horizon 2020 research and innovation programme under the Marie Sklodowska-Curie grant agreement No. 721732. (Corresponding author: Adrián Lahuerta-Lavieja).

A. Lahuerta-Lavieja and G. A. E. Vandenbosch are with the Department of Electrical Engineering, KU Leuven, 3001 Leuven, Belgium (e-mail: adrian.lahuerta@kuleuven.be; guy.vandenbosch@kuleuven.be).

M. Johansson and U. Gustavsson are with Ericsson Research, Ericsson AB, 41756 Gothenburg, Sweden (e-mail: martin.n.johansson@ericsson.com; ulf.gustavsson@ericsson.com).

T. A. H. Bressner is with the Department of Electrical Engineering, Eindhoven University of Technology, 5600MB Eindhoven, Netherlands (email: t.a.h.bressner@tue.nl).

The MATLAB code used in the generation of some of the figures is available on GitHub: https://github.com/adrlah/singleScattering. provision of reliable and accurate mm-wave channel models is key for supporting the deployment of ultra-high capacity $5 \mathrm{G}$ networks [3]. With this vision, extensive mm-wave radiochannel measurement campaigns have been carried out worldwide in various propagation environments and at multiple frequencies during the last decade. COST2100, METIS, COST IC1004, mmMAGIC, and NYU WIRELESS are some of the most relevant consortiums and research groups that have been leading the mm-wave band measurement and modeling efforts.

COST 2100 [4], in agreement with [5], [6], emphasizes the importance of diffuse scattering at mm-wave frequencies, especially in non-line-of-sight (NLoS) scenarios. However, METIS measurement results indicate that specular paths may dominate in many scenarios and, therefore, suggest that $5 \mathrm{G}$ channel schemes using steerable highly directive antennas should take into account realistic modelling of specular paths [7]. Furthermore, COST IC1004 suggests independent temporal and spatial clustering in mm-wave channels due to a richer scattering for shorter wavelengths [8], [9], while mmMAGIC stresses the importance of characterizing blocking and scattering objects in a proper way [3]. Measurements of specular and diffuse scattering from different types of surfaces were performed in the context of the mmMAGIC project [10].

In terms of blockage, METIS proposes [7, Sec. C.1.4] the use of a three-dimensional (3D) version of the multiple knife-edge diffraction (MKED) model presented in [11]. This model evaluates the arctangent function with an argument proportional to the excess path length of the rays traveling around the body edges. Following METIS, mmMAGIC proposes an improved version of the METIS blockage model by introducing shadow zone diffraction loss and terms accounting for the phase variations of the paths around the edges [12, Sec. 4.6.3]. This model has been included in International Telecommunication Union (ITU) Recommendation P.526-14 as a "semi-empirical" (SE) model with a small numerical approximation [13, Sec. 5.2.1.2].

In addition, body diffraction models based on the Fresnel integrals have been proposed. A two-dimensional knife-edge model for obstruction of a ray by a person was presented in [14]. A similar approach for received signal strength (RSS) variation due to body diffraction was introduced in [15]. In this approach, the target is regarded as a cylinder and diffraction is only considered along the width dimension of the object. The parameter evaluated in the Fresnel integrals is again a function of the excess path length due to diffraction around the edges. A somewhat less intuitive 3D Fresnel-integral-based formulation, which captures the excess path length only implicitly, was 
presented in ITU Recommendation P.526-14 [13, Sec. 5.2.1.1]. This ITU Fresnel approach is only valid for small angles between the surface normal vector and the line drawn between transmit (Tx) and receive (Rx) antennas. Finally, the models of [7, Sec. C.1.4] and [15] have been extended with antenna gains towards the edges in [16] and [17], respectively.

Using the radar cross section (RCS) is a standard approach for representing an object's ability to scatter a signal in the direction of a receiver. Although RCS assumes isotropic scattering at the object, when the plate dimension is much greater than the wavelength, the RCS of the conducting plate observed at the normal direction can be approximated by the product of the physical area and the effective gain of the plate [18]. To this extent, a mm-wave backscattering model based on the RCS of a sphere was also proposed by METIS [7, Sec. C.1.5]. Despite the computational speed of RCS models, these solutions are only accurate in the far-field, as will be illustrated in Section III.

Another option for calculating backscattered fields are raytracing (RT) or ray-based methods. RT solutions, see, e.g. [19], [20], are commonly based on the Uniform Theory of Diffraction (UTD) and Fresnel reflection coefficients [21, pp. 179-180], [22, pp. 9-10]. These solutions are very complete (they may include diffuse scattering, polarization information, etc.) yet computationally-heavier than the previously introduced forward-scattering models. In this paper, we attempt to fill the gap of low-complexity backscattering models from rectangular screens whose accuracy could compete with raybased approaches.

Regarding full-channel modeling, geometry-based stochastic channel models (GSCMs) have been proposed as one of the most suitable candidates at mm-wave frequencies [23]. They combine the advantages of geometry-based models (they can be seen as a simplified ray tracing approach) with the advantages of stochastic models (various carrier frequencies, realistic large scale and small scale parameter correlations, and dynamic modeling are supported). Nevertheless, knowledge of the scenario geometry, i.e., distances, heights and orientations of the objects is required. GSCM implementation is commonly based on random deployment of scatterers in the scenario according to a certain distribution, assignment of different channel properties and assessment of their respective contribution to the received signal. For example, the integration of the METIS blockage model [7, Sec. C.1.4] in a complete GSCM in the context of $5 \mathrm{G}$ communications has been published in [24].

In this paper, we present computationally-efficient scalar scattering models for the backscattered field from planar smooth rectangular scattering surfaces. First, the Fresnelintegrals-based model from [15] is extended to 3D for arbitrary object orientation. In addition, a simpler but less accurate model based on the error function is also presented as an approximation of the scattering coefficient when the specular reflection point lies within the surface of the object. Second, the aforementioned blockage models (METIS [7, Sec. C.1.4], mmMAGIC [12, Sec. 4.6.3]/ITU SE [13, Sec. 5.2.1.2], and ITU Fresnel [13, Sec. 5.2.1.1]), originally defined to characterize body shadowing phenomena, are re-formulated to account for the backscattered field from a surface by applying the ray optical continuation at high frequencies. Furthermore, for all models, only a single point on the scattering surface is chosen for computing antenna gains and wave phase delay as opposed to other works that use four [16], [17]. Finally, the performance of the re-formulated, extended, and proposed models are validated against electromagnetic (EM) simulations and measurements.

The models proposed in Section II capture the backscattered fields from a smooth rectangular surface both in the nearfield and in the far-field. Since the models only use one point on the scattering surface, one of the expected results would be their usage in studies based on random point-scatterer deployment such as GSCM-based simulators. The majority of investigations in the literature are based on point-scatterer models which describe the scatterers' total contribution with a fading magnitude distribution and a uniformly-distributed random phase. This assumption is motivated using the central limit theorem when the number of scatterers tends to infinity [25]-[27]. Nevertheless, this assumption might be unrealistic in sparse mm-wave channels dealing with real environments where the specular reflection point does not lie on the surface of finite objects/scatterers. Therefore a model exploiting the real scenario geometry, i.e., location, orientation, and size of the scattering objects, is important and will be of use for producing more realistic GSCM-based simulations. For instance, generating random rectangular plates with different size, orientation, and location instead of point-scatterers may more realistically mimic daily environments and actual cluttering objects such as billboards, vehicles, or even human bodies [11], [15].

Another, more general, value of the proposed models is as a complement to more detailed EM models, such as physical optics and rigorous full-wave models. The proposed models offer a first-order approximation with minimal conceptual and computational complexity, which makes them easy to implement and allows for their use in exploratory studies, for example in conjunction with optimization tools. The proposed models are well suited for use in link and systems simulators used for evaluation of multiple-input multiple-output (MIMO) performance. Such problems typically involve very many, hundreds or more, large objects (in terms of wavelengths), which makes rigorous EM modeling impossible or prohibitively timeconsuming.

The remainder of the paper is structured as follows. Section II introduces the proposed single-scattering models. The models are validated by means of simulations and measurements in Section III. Finally, Section IV concludes this paper.

\section{Single-SCATtering Model}

In agreement with measurement results, the channel response can be described as the addition of four different contributions: "(i) a line-of-sight (LoS) component, (ii) discrete components due to reflections on mobile scatterers, (iii) discrete components arising due to reflections on static scatterers, and (iv) diffuse components" [28]. The focus of this paper is on modeling Tx-to-Rx propagation in the presence of 


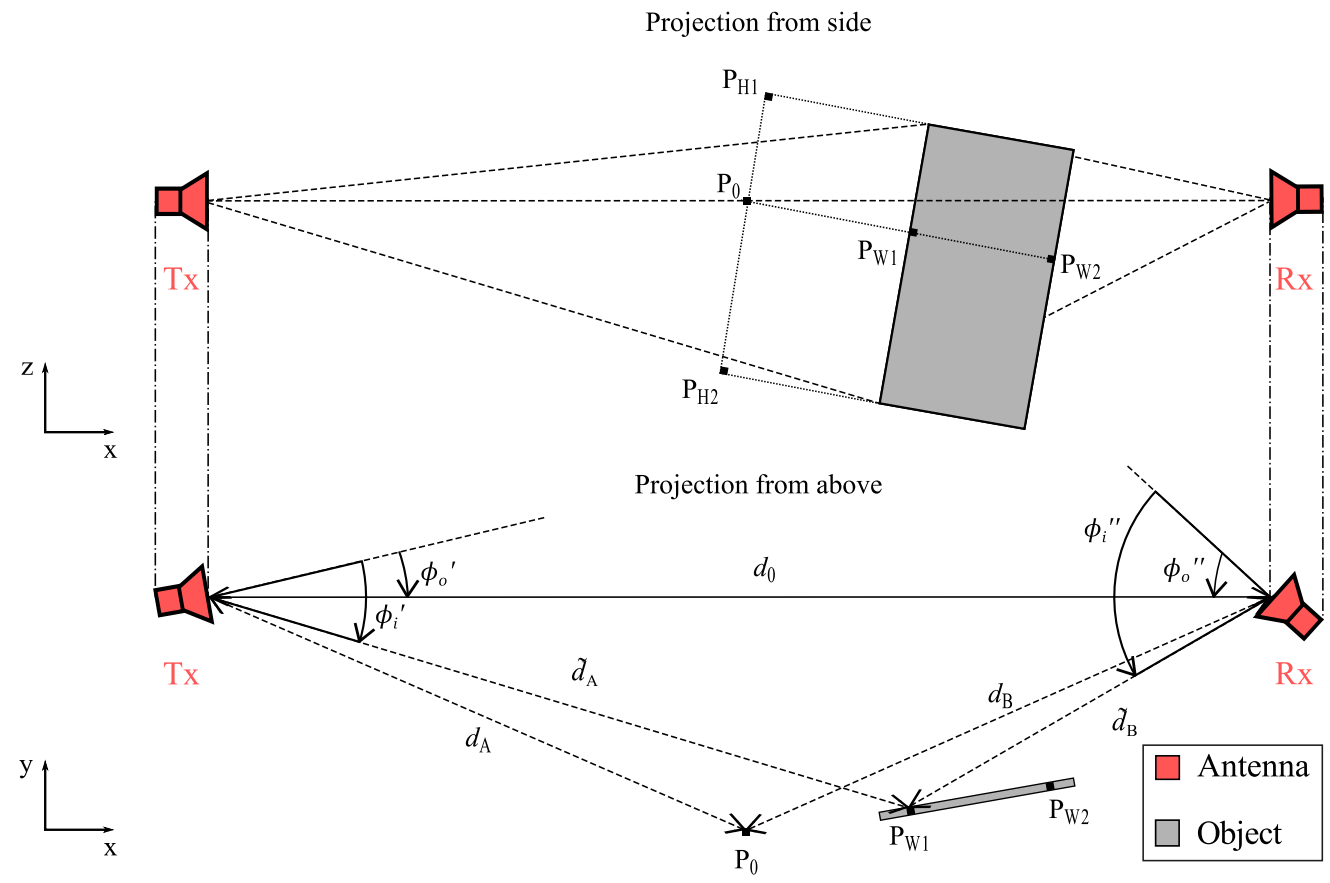

Fig. 1. Example scenario with the antennas located on the $\mathrm{x}$-axis and a scattering surface in a plane parallel to the xz-plane: (top) side projection and (bottom) top projection.

single-scattering contributions from static scatterers, although mobile scatterers can also be modeled adding time variability. Therefore, the channel response $h_{i}$ due to the $i$ th static scatterer can be written as

$$
\begin{aligned}
h_{i} & =h\left(\mathbf{d}_{\mathrm{Tx}}, \mathbf{R}_{\mathrm{Tx}}, \mathbf{d}_{\mathrm{Rx}}, \mathbf{R}_{\mathrm{Rx}}, \mathbf{d}_{\mathrm{SS}_{i}}, \mathbf{R}_{\mathrm{SS}_{i}}\right) \\
& =h\left(\Omega_{\mathrm{Tx}_{i}}, \Omega_{\mathrm{Rx}_{i}}, d_{i}\right)
\end{aligned}
$$

where $\Omega_{\mathrm{Tx}_{i}}$ is the direction of departure from the Tx antenna in the Tx antenna coordinate system, $\Omega_{\mathrm{Rx}_{i}}$ is the direction of arrival at the Rx antenna in the Rx antenna coordinate system, and $d_{i}$ is the distance from the Tx antenna to the Rx antenna via the specular reflection point $\mathrm{P}_{0}$ in the plane defined by the $i$ th scatterer, e.g. $d=d_{\mathrm{A}}+d_{\mathrm{B}}$ for the scatterer in Fig. 1 . The variables $\Omega_{\mathrm{Tx}_{i}}, \Omega_{\mathrm{Rx}_{i}}$, and $d_{i}$ can be derived from globalcoordinates variables $\mathbf{d}_{\mathrm{Tx}}, \mathbf{d}_{\mathrm{Rx}}, \mathbf{d}_{\mathrm{SS}_{i}}, \mathbf{R}_{\mathrm{Tx}}, \mathbf{R}_{\mathrm{Rx}}$, and $\mathbf{R}_{\mathrm{SS}_{i}}$ : $\mathbf{d}_{\mathrm{Tx}}, \mathbf{d}_{\mathrm{Rx}}$, and $\mathbf{d}_{\mathrm{SS}_{i}}$, denote the displacement vectors of the Tx antenna, Rx antenna and the $i$ th scatterer, respectively; $\mathbf{R}_{\mathrm{Tx}}$, $\mathbf{R}_{\mathrm{Rx}}$, and $\mathbf{R}_{\mathrm{SS}_{i}}$, are the orientations of the Tx antenna, $\mathbf{R x}$ antenna and the $i$ th scatterer, respectively.

The total channel response $h$ in the presence of $N$ static scatterers is written as

$$
h=\underbrace{h\left(\Omega_{\mathrm{Tx}_{0}}, \Omega_{\mathrm{Rx}_{0}}, d_{0}\right)}_{h_{0}}+\sum_{i=1}^{N} \underbrace{h\left(\Omega_{\mathrm{Tx}_{i}}, \Omega_{\mathrm{Rx}_{i}}, d_{i}\right)}_{h_{i}}
$$

where subscript 0 denotes the LoS component, hence $d_{0}$ is the Tx-Rx LoS distance. To facilitate readability, the majority of the variables defined in the following are illustrated in Fig. 1 for Tx and Rx antennas located at the same height. First, the
LoS component $h_{0}$ is defined as

$$
\begin{aligned}
h_{0} & =h\left(\Omega_{\mathrm{Tx}_{0}}, \Omega_{\mathrm{Rx}_{0}}, d_{0}\right) \\
& =g_{\mathrm{Tx}}\left(\theta_{0}^{\prime}, \phi_{0}^{\prime}\right) g_{\mathrm{Rx}}\left(\theta_{0}^{\prime \prime}, \phi_{0}^{\prime \prime}\right) \frac{\lambda}{4 \pi} \frac{e^{-j k d_{0}}}{d_{0}}
\end{aligned}
$$

where $g_{\mathrm{Tx}}$ and $g_{\mathrm{Rx}}$ are the Tx and Rx antenna gains $\left(g_{\mathrm{Tx}}=\right.$ $\sqrt{G_{\mathrm{Tx}}}$ and $g_{\mathrm{Rx}}=\sqrt{G_{\mathrm{Rx}}}$ where $G_{\mathrm{Tx}}$ and $G_{\mathrm{Rx}}$ are the linear power gains), respectively, $\theta_{0}^{\prime}$ and $\phi_{0}^{\prime}$ are elevation and azimuth angles, respectively, towards the Rx antenna in the Tx local coordinate system, $\theta_{0}^{\prime \prime}$ and $\phi_{0}^{\prime \prime}$ are elevation and azimuth angles, respectively, towards the Tx antenna in the Rx local coordinate system, and $k=2 \pi / \lambda$ is the wavenumber, with $\lambda$ being the wavelength. The factor $\lambda / 4 \pi$ is included to take into account the power density of transmitted field and the effective area of the Rx antenna. Second, the contribution of the $i$ th scatterer $h_{i}$ is written as

$$
h_{i}=g_{\mathrm{Tx}}\left(\theta_{i}^{\prime}, \phi_{i}^{\prime}\right) \Gamma_{i} g_{\mathrm{Rx}}\left(\theta_{i}^{\prime \prime}, \phi_{i}^{\prime \prime}\right) \frac{\lambda}{4 \pi} \frac{e^{-j k d_{i}}}{d_{i}}
$$

where the scalar scattering coefficient $\Gamma_{i}=\Gamma_{i}^{\mathrm{G}} \cdot \Gamma_{i}^{\mathrm{F}}$ models the effect of the scattering process on the wave scattered in the direction of the receiving antenna, i.e., geometry and electrical properties of the $i$ th scatterer. Here, the size and orientation of the $i$ th scatterer are captured by the geometrical scattering coefficient $\Gamma_{i}^{\mathrm{G}}$ and the material properties of the scatterer are based on the Fresnel reflection coefficients $\Gamma_{i}^{\mathrm{F}}[21$, pp. 179180], [22, pp. 9-10]. $\Gamma_{i}^{\mathrm{G}}$ is assumed separable and calculated as

$$
\Gamma_{i}^{\mathrm{G}}=\Gamma_{i}^{\mathrm{W}} \cdot \Gamma_{i}^{\mathrm{H}},
$$

where $\Gamma_{i}^{\mathrm{W}}$ and $\Gamma_{i}^{\mathrm{H}}$ are the independent scattering coefficients calculated in the width and height dimensions of the scattering 
rectangle, respectively. The calculation of $\Gamma_{i}^{\mathrm{G}}$ is the goal of this paper and will be assessed by different models.

Furthermore, $\theta_{i}^{\prime}$ and $\phi_{i}^{\prime}$ are elevation and azimuth angles, respectively, towards the antenna gain reference point in the $i$ th scattering surface in the Tx local coordinate system, $\theta_{i}^{\prime \prime}$ and $\phi_{i}^{\prime \prime}$ are elevation and azimuth angles, respectively, towards the antenna gain reference point in the $i$ th scattering surface in the $\mathrm{Rx}$ local coordinate system. The antenna gain reference point is the point used to compute antenna gains. This point is either the specular reflection point, if contained within the $i$ th scattering surface, or the point in the scattering surface closest to the specular reflection point in the plane of the scattering surface. For example, the antenna gain reference point in the scenario depicted in Fig. 1 is $\mathrm{P}_{\mathrm{W} 1}$. Moreover, $\mathrm{P}_{\mathrm{W} 1}$ and $\mathrm{P}_{\mathrm{H} 1}$ denote the points on the line defined by the closest edges to $\mathrm{P}_{0}$ in the width and height dimensions, respectively, and $\mathrm{P}_{\mathrm{W} 2}$ and $\mathrm{P}_{\mathrm{H} 2}$ are points on the furthest edges.

The ray optical continuation at high frequencies [29] has been applied in (4), i.e., the divergence factor or spreading factor governs the amplitude variation of the geometrical optics field along the ray path. Equation (4) is fundamentally based on considering the three natural steps in the scattering process: the propagation of the wave from the Tx antenna to the scatterer, the scattering at the finite scatterer, and the propagation of the wave from the scatterer to the $\mathrm{Rx}$ antenna. The novelty of the presented approach for calculating the scattering is that it includes the finiteness of the scatterer while keeping the point-scatterer perspective when it comes to the wave's phase delay, i.e., the GSCM structure is preserved. This is clearly an approximation since not only one point but the whole object is radiating due to the EM scattering phenomena. This approximation is more accurate at high frequencies, as the object's size becomes larger with respect to the wavelength, making a ray optic model tractable and full-wave simulations less affordable. Nevertheless, this is complemented by a model which takes into account the whole geometry of the scenario to assess the scatterers contribution to the total received field, i.e., position and orientation of the antennas, and position, orientation, and size of the scatterers.

The model is scalar and hence does not include effects related to the polarization state of the incident field, approximating the scattering from finite surfaces using only the incident field over the surface as the basis for the scattered field, rather than the actual induced currents, i.e., the field is (implicitly) abruptly truncated at the edges. Furthermore, the model assumes LoS to the scattering object for both Tx and Rx antennas, i.e., it is a single-scattering model for surfaces visible from both antennas. Finally, the model is also reciprocal.

To model the scattering from a semi-infinite half-screen, we use the scalar knife edge diffraction (KED) solution [30], [31]. According to Fresnel-Huygens principle, the field strength of the diffracted field is proportional to [32]

$$
\int_{\nu}^{\infty} \exp \left(-j \frac{\pi t^{2}}{2}\right) d t
$$

where $\nu$ denotes the Fresnel-Kirchhoff diffraction parameter. Using the Fresnel integrals, $\mathrm{C}(\nu)=\int_{0}^{\nu} \cos \left(\frac{\pi t^{2}}{2}\right) d t$ and

$$
\begin{aligned}
& \mathrm{S}(\nu)=\int_{0}^{\nu} \sin \left(\frac{\pi t^{2}}{2}\right) d t, \text { and } \\
& \mathrm{F}(\nu)=\mathrm{C}(\nu)-j \mathrm{~S}(\nu)=\int_{0}^{\nu} e^{-j \frac{\pi}{2} t^{2}} d t,
\end{aligned}
$$

the expression in (6) can be written as

$$
\int_{\nu}^{\infty} \exp \left(-j \frac{\pi t^{2}}{2}\right) d t=\mathrm{F}(0)-\mathrm{F}(\nu) .
$$

The Fresnel-Kirchhoff diffraction parameter $\nu$ is defined as [15]

$$
|\nu|=2 \sqrt{\frac{\Delta d}{\lambda}}
$$

where $\Delta d$ denotes the excess path length via the edge of the half-screen with respect to the length of the shortest path, i.e., the path via the specular reflection point $\mathrm{P}_{0}$ when transmitter and receiver are on the same side of the plane defined by the half-screen.

The expression in (8) is valid for a half-screen. In the case of a strip, the total field of the diffracted signals is calculated by addition of the two individual edges [14, eq. (5)]. In our work, we use this procedure for each pair of parallel edges of finite rectangular scatterers. Hence, in our model, we use (7) to calculate the scattering coefficients $\Gamma_{i}^{\mathrm{W}}$ and $\Gamma_{i}^{\mathrm{H}}$ related to the dimensions of the rectangular scattering object.

For the example scenario depicted in Fig. 1, in the case of the closest edge point to $\mathrm{P}_{0}$ in the width dimension, $\mathrm{P}_{\mathrm{W} 1}$, we have that the excess path length via this point is $\Delta d_{\mathrm{W} 1}=\tilde{d}_{\mathrm{A}}+\tilde{d}_{\mathrm{B}}-d$, and thus $\nu_{\mathrm{W} 1}$ can be computed from (9). The same procedure is to be applied to the other edge points $\mathrm{P}_{\mathrm{W} 2}, \mathrm{P}_{\mathrm{H} 1}$ and $\mathrm{P}_{\mathrm{H} 2}$ for calculating $\nu_{\mathrm{W} 2}, \nu_{\mathrm{H} 1}$, and $\nu_{\mathrm{H} 2}$, respectively. Although the calculation of each $\nu_{i}$ is individual, the diffraction parameters are applied for pairs of parallel edges as shown below.

The same reasoning can be applied to the blockage models (METIS [7, Sec. C.1.4], mmMAGIC [12, Sec. 4.6.3], ITU Fresnel [13, Sec. 5.2.1.2], and ITU Semi-empirical [13, Sec. 5.2.1.1]), introduced in Section I, by applying the ray optical continuation as was done in (4). Reformulated versions of these models are proposed to be used to account for backscattered field.

In addition, the error function $\operatorname{erf}(x)$ is also considered in this study as a magnitude-only approximation to (7). The error function $\operatorname{erf}(x)$ is defined as

$$
\operatorname{erf}(\nu)=\frac{2}{\sqrt{\pi}} \int_{0}^{\nu} e^{-t^{2}} d t
$$

Generally, the proposed scattering coefficient (in the width dimension of the scattering rectangle) $\Gamma^{\mathrm{W}}$ is calculated as

$$
\Gamma^{\mathrm{W}}= \begin{cases}K_{m}\left[f_{m}\left(\nu_{\mathrm{W} 2}\right)+f_{m}\left(\nu_{\mathrm{W} 1}\right)\right] & \mathrm{P}_{0} \text { betw. } \mathrm{P}_{\mathrm{W} 1} \text { and } \mathrm{P}_{\mathrm{W} 2} \\ K_{m}\left[f_{m}\left(\nu_{\mathrm{W} 2}\right)-f_{m}\left(\nu_{\mathrm{W} 1}\right)\right] & \text { otherwise }\end{cases}
$$

where $f_{m}$ denotes the function among the proposed models as indicated in Table I, $\nu_{\mathrm{W} 1}$ and $\nu_{\mathrm{W} 2}$ are the Fresnel-Kirchhoff diffraction parameters due to the excess path length from the Tx antenna to the $\mathrm{Rx}$ antenna via $\mathrm{P}_{\mathrm{W} 1}$ and $\mathrm{P}_{\mathrm{W} 2}$, respectively, and $K_{m}$ is a complex normalization constant such that the scattering coefficient $\Gamma^{\mathrm{W}} \rightarrow 1$ asymptotically as the arguments 
TABLE I

MODEL FUNCTIONS

\begin{tabular}{cccc}
\hline Model $(m)$ & $f_{m}(\cdot)$ & & $K_{m}$ \\
\cline { 1 - 1 } 3D Fresnel & $(7)$ & & $j / 2$ \\
erf & & $(10)$ & \\
M-METIS & & [7, eq. (C-8)] & \\
M-ITU SE & & {$[13$, eq. (75)]] } & \\
M-ITU Fresnel & {$\left[13\right.$, eq. (72)-(73)] ${ }^{1}$} & & $j / 2$ \\
\hline
\end{tabular}

$\nu_{\mathrm{W} 1}$ and $\nu_{\mathrm{W} 2}$ tend to $\infty$, i.e., an infinitely wide surface; see Table I. The same reasoning applies along the height dimension for calculating $\Gamma^{\mathrm{H}}$.

Finally, the scattering coefficient $\Gamma$ is calculated by multiplication of the geometrical scattering coefficient $\Gamma^{\mathrm{G}}$, see (5), obtained following the principles described in this paper, and the Fresnel reflection coefficient $\Gamma^{\mathrm{F}}$. The scattering coefficient $\Gamma$ thus captures the effect of the location, orientation, size, and electrical properties of the scatterer.

\section{RESULTS}

In order to demonstrate the validity of the proposed models at mm-waves, a performance comparison against more complete simulation models and measurements is presented in this section. The main carrier frequency used here is 28 $\mathrm{GHz}$, although simulation results at 39 and $60 \mathrm{GHz}$ are also presented, and all scatterers are assumed to be perfect electric conductors (PEC), i.e., $\Gamma^{\mathrm{F}}=-1$. Furthermore, the verticallypolarized, i.e., z-polarized,) antennas used in the simulations (unless stated otherwise) are models of the ones used in [33], Schwarzbeck 9170 and Narda V637 for the Tx and Rx antenna, respectively, scaled with the wavelength to have the same radiation properties at all studied frequencies. Finally, although all the discussed models have been implemented and tested in each scenario, in some figures only the 3D Fresnel results are shown for the sake of readability, since this model has the best performance (as will be shown in the following via statistical error metrics).

\section{A. Validation Against EM Simulations}

GRASP is a well-known and validated software developed by TICRA for reflector antennas. The current version of this software supports full-wave simulations using the Method of Moments (MoM) [34], in addition to PO, with the possibility of extending the latter with the Physical Theory of Diffraction (PTD).

To test the simple scattering model, a scenario consisting of a $0.3 \times 0.5$-meter rectangle was simulated at $28 \mathrm{GHz}$ in GRASP. The rectangle centroid is located at the origin $\mathrm{O}$, denoted by a green dot in Fig. 2(a). In this example, an isotropic Tx antenna has been used to provide comparable power levels at the Rx antenna due to the direct and the scattered-from-the-rectangle components while the $\mathrm{Rx}$ antenna is rotated in the $[-90,+180]$ degree interval.

\footnotetext{
${ }^{1}$ ITU Fresnel [13, Sec. 5.2.1.2] uses a diffraction parameter that captures the excess path length only implicitly.
}

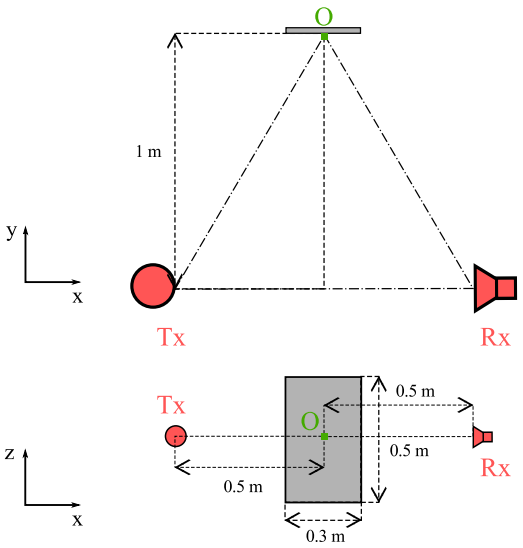

(a)

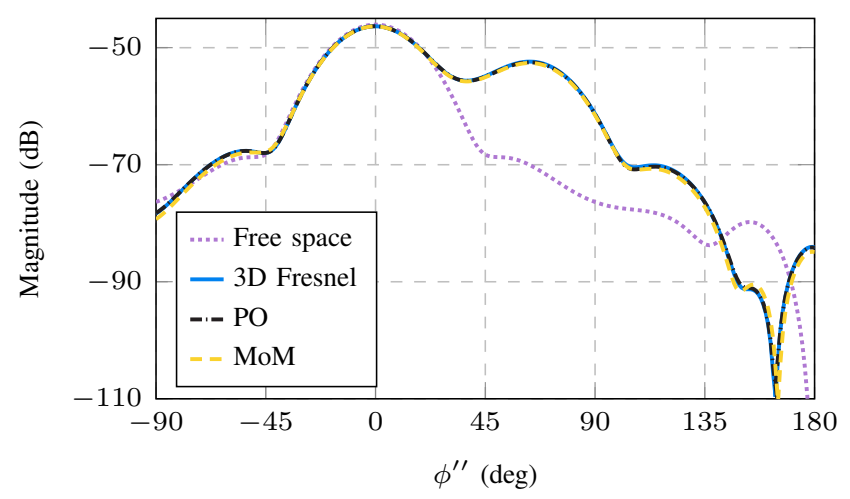

(b)

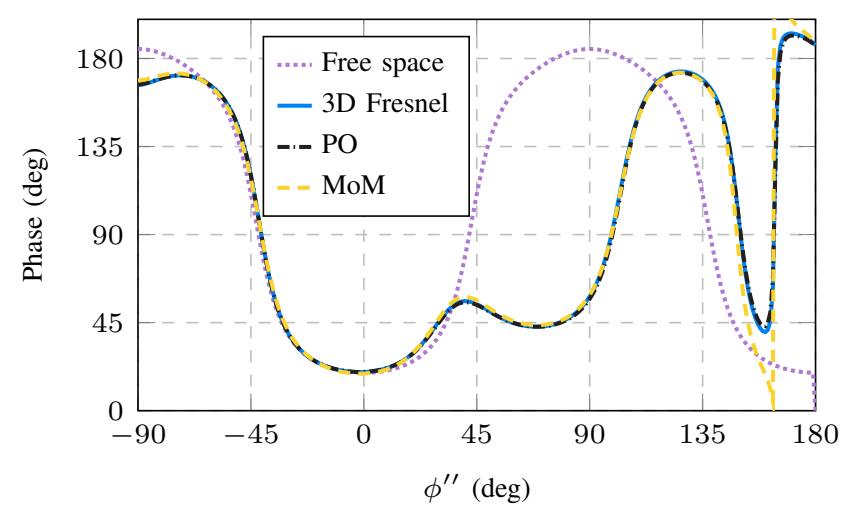

(c)

Fig. 2. Test-case with a PEC rectangle and a Tx isotropic radiator at 28 GHz. (a) From left to right: top and side projections, respectively, of the case representing 0-degree Rx antenna pointing; (b) magnitude and (c) phase results as a function of the Rx antenna pointing direction $\phi^{\prime \prime}$.

The results obtained for the MoM, PO combined with PTD, and the proposed 3D Fresnel-integrals model are presented in Figs. 2(b) and 2(c). The first conclusion drawn from them is that, at these frequencies, the MoM full-wave simulation and the $\mathrm{PO}$ approximation yield very similar performance and thus $\mathrm{PO}$ will be used as a reference in the remainder of the paper for comparison with the presented model results. Furthermore, it can be also observed that the proposed model yields a good agreement with the PO and MoM results. Nevertheless, simulation-time and complexity differences are extreme, with the 3D Fresnel model providing more than two to four orders 
TABLE II

COMPUTATION TIME AND COMPLEXITY OF THE DIFFERENT MODELS

\begin{tabular}{|c|c|c|}
\hline Method & Computation time & Complexity \\
\hline MoM & 30 minutes & $\mathcal{O}(N \log N)[35]$ \\
\hline PO with PTD & 20 seconds & $\mathcal{O}(N)[36]$ \\
\hline Proposed models & 0.15 seconds & $\mathcal{O}(1)$ \\
\hline
\end{tabular}

of magnitude faster results than the MoM and PO conventional models, as shown in Table II -where $\mathcal{O}$ and $N$ denote big-O notation and the number of unknowns, respectively-, in spite of the Fresnel model being implemented in MATLAB. Even accounting for potential acceleration techniques for the MoM and PO models, there will still be orders of magnitude difference in simulation-time, simply because of the formulation of the solution, where the complexity and simulation time of the proposed 3D Fresnel model is independent of the size of the scattering surface.

\section{B. Performance Comparison of the Models}

The METIS [7, Sec. C.1.4] and ITU semi-empirical (ITU SE) [13, Sec. 5.2.1.2] blockage models, the latter also presented as the mmMAGIC model [12, Sec. 4.6.3], have been proposed for assessing the diffraction loss by rectangular screens. Both of these models make use of the arctangent (atan) function as their basis to calculate the diffraction loss. Similarly to the error-function model proposed here, the limitation of this approach is that it does not capture phase variations. For that reason, the ITU SE/mmMAGIC model introduces phase terms to the scattering-surface edges to consider phase changes. In addition, the ITU Fresnel model [13, Sec. 5.2.1.1] makes use of the Fresnel integrals to solve the same problem and accounts for both magnitude and phase behavior. Nevertheless, as will be shown in this section, these models can be re-used to account for backscattering from rectangular surfaces following the reasoning proposed in this paper.

Fig. 3 shows the behavior of the three functions which form the core of the models as listed above. The functions have been normalized to $\sqrt{K_{m}}$, where $K_{m}$ is the normalization constant for each model, see Table I. In addition, the argument of the arctangent function has been scaled according to [11].

Fig. 3(a) shows the magnitude oscillating behaviour of the complex Fresnel integral function F in (7) and the monotonic growth of the arctangent and error functions. Furthermore, the error-function model approximates (asymptotically) the mean of the Fresnel expression, over one period of oscillation, whereas the arctangent function, used by the METIS and ITU SE/mmMAGIC models, traces the lower envelope of the magnitude of the Fresnel integral. Fig. 3(b) illustrates the phase dependence of the function $F$ function, whereas the error and arctangent functions do not capture phase variation.

In the following, four different scenarios are used to test the models proposed in Section II against PO results. The scenarios were chosen to evaluate the performance of the models for different angles of incidence and departure, orientations, and distances with respect to the scattering surface.

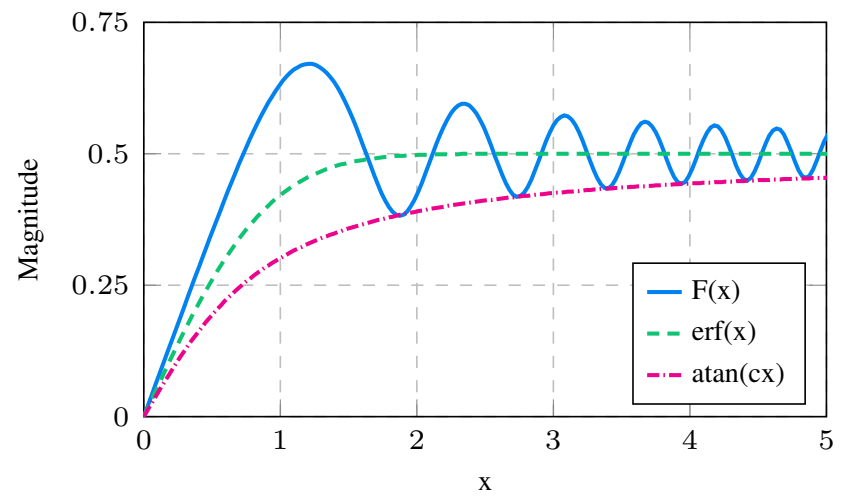

(a)

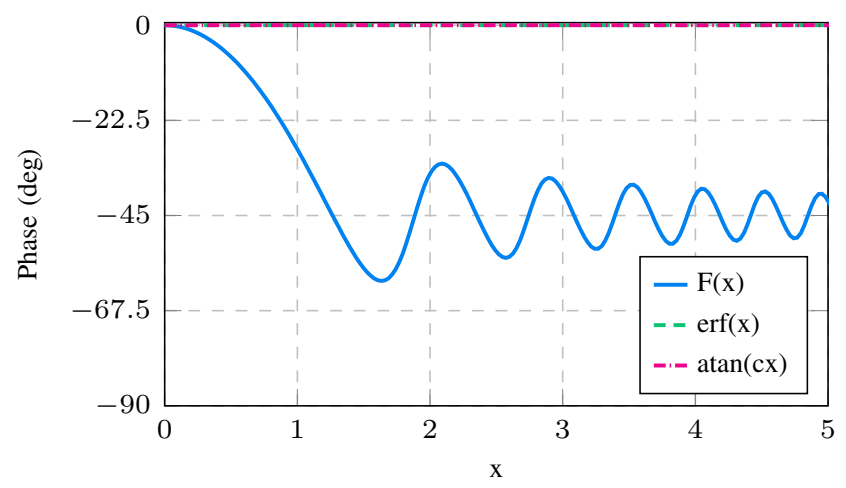

(b)

Fig. 3. (a) Magnitude and (b) phase behavior of the F, error, and arctangent functions.

The performance is assessed in terms of both plots and error metrics.

The statistical error metrics used are the normalized meansquare error (NMSE) and the weighted normalized meansquare error (WNMSE). The latter metric has been introduced for compensating the quadratic power decay with distance in the last scenario, Scenario D. The NMSE is defined as [37]

$$
\mathrm{NMSE}=10 \log _{10}\left(\frac{\sum_{n}\left|x_{n}-\hat{x}_{n}\right|^{2}}{\sum_{n}\left|x_{n}\right|^{2}}\right)
$$

where $x$ denotes the complex reference signal, i.e., the PO simulations, and $\hat{x}$ the complex estimated signal, i.e. the proposed models. The WNMSE is defined as

$$
\mathrm{WNMSE}=10 \log _{10}\left(\frac{\sum_{n} w_{n}\left|x_{n}-\hat{x}_{n}\right|^{2}}{\sum_{n} w_{n}\left|x_{n}\right|^{2}}\right)
$$

where $w$ is the vector of weights. The weighting vector $w_{n}$ for Scenario $\mathrm{D}$ is chosen to be $w_{n}=d_{n}^{2}$, where $d_{n}$ denotes the distance from the Rx antenna to the rectangle centroid at sample $n$.

As mentioned previously, the state-of-the-art blockage models are modified in this paper to capture backscattered fields. To avoid confusion with their original purpose and definition, the proposed modified versions will be denoted in the following with a distinctive "M-" (for modified) prior to their name.

First, Scenario A evaluates the performance of the models for different orientations of the scatterer. Hence, a "rotating 


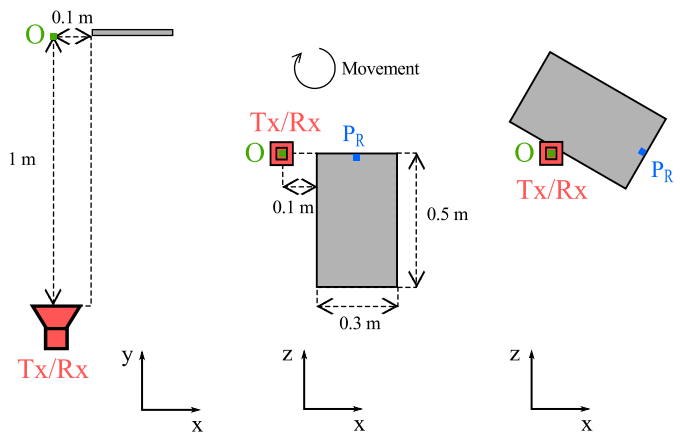

(a)

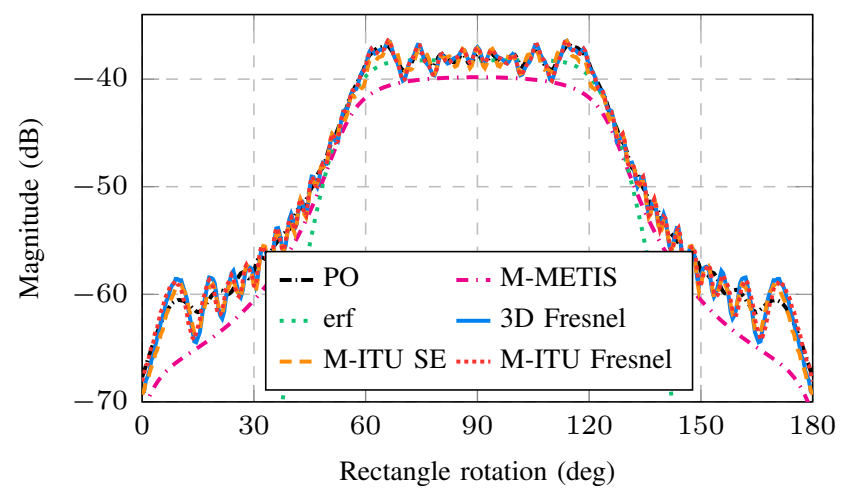

(b)

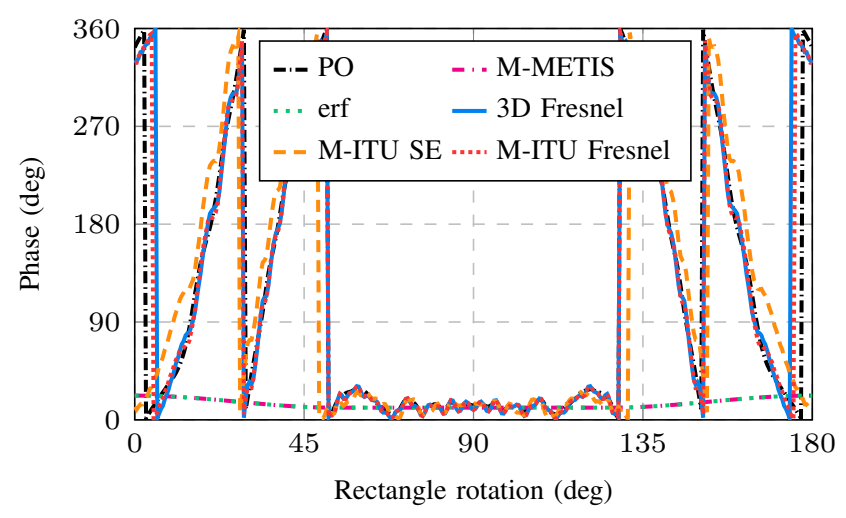

(c)

Fig. 4. Scenario A: normal-incidence rotating rectangle at $60 \mathrm{GHz}$. (a) From left to right: top projection and side projection for 0 - and 120-degree rotation around $\mathrm{P}_{\mathrm{R}}$, respectively; (b) magnitude and (c) phase results for different rectangle rotations.

rectangle" geometry was designed, see Fig. 4(a). The $0.3 \times 0.5-$ meter $\mathrm{PEC}$ rectangle is rotated clockwise around the rotation point $P_{R}$ with start and end points at 0 (the rectangle extending downwards in the figure) and 180 degrees, respectively. This is performed at $60 \mathrm{GHz}$ for collocated Tx and Rx antennas at one meter distance from the plane of the rectangle, the antennas pointing to the origin $\mathrm{O}$, i.e., normal incidence to the rectangle plane as shown in Fig. 4(a). The M-ITU models, both SE and Fresnel, and the herein proposed 3D Fresnel model show good agreement when compared to the PO reference both in magnitude and phase, see Figs. 4(b) and 4(c). Moreover, the performance of the M-ITU Fresnel model and the herein presented 3D Fresnel model is almost identical in this normal-

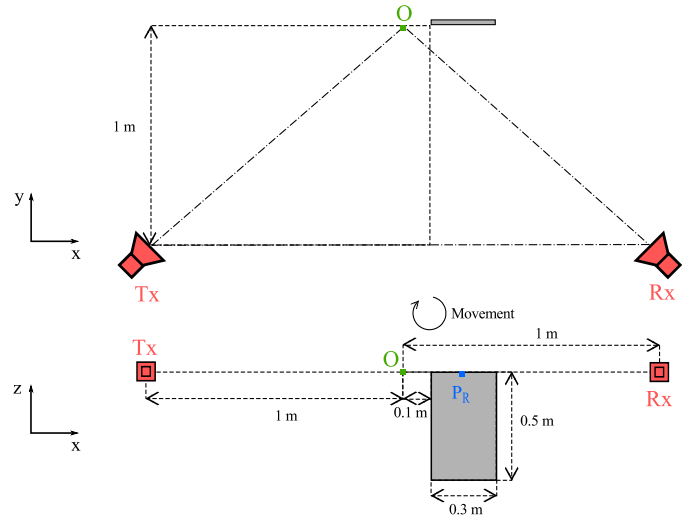

(a)

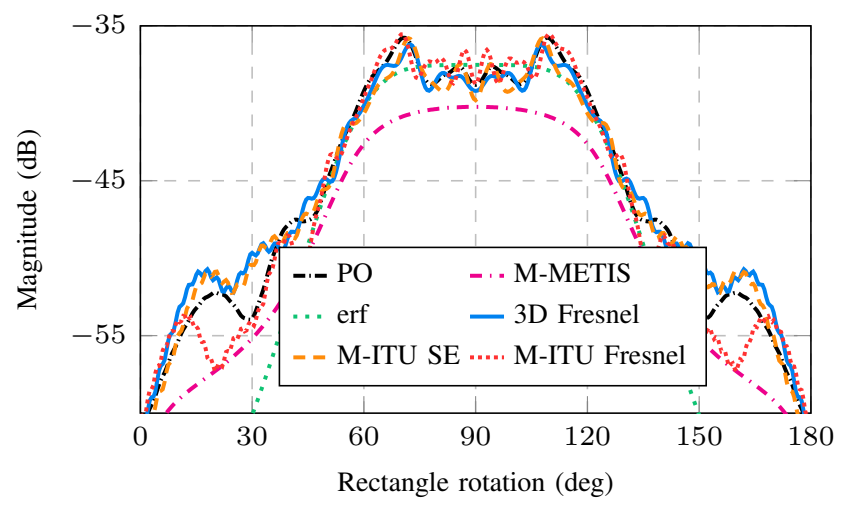

(b)

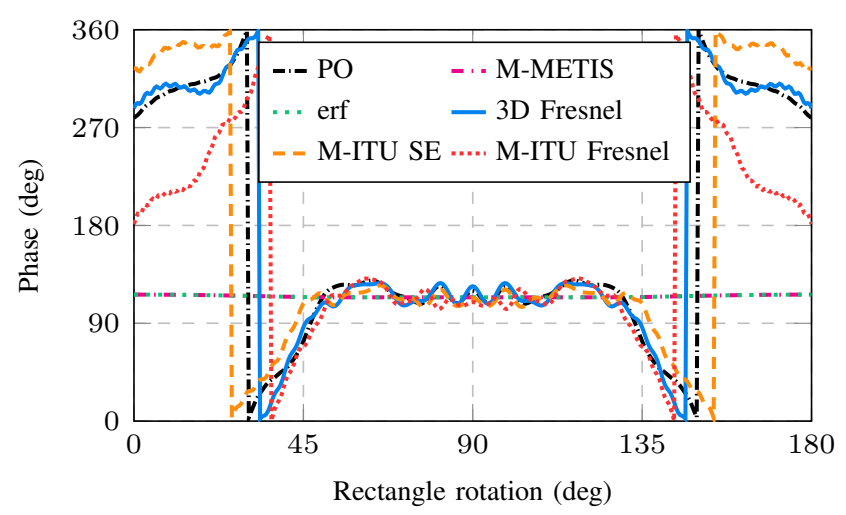

(c)

Fig. 5. Scenario B: oblique-incidence rotating rectangle at $39 \mathrm{GHz}$. (a) (Top) top projection and (bottom) side projection for 0-degree surface rotation; (b) magnitude and (c) phase results for different rectangle rotations.

incidence case, see Table III. The erf model seems to capture the average of the other models while the M-METIS model traces the lower envelope, which coincide with the behavior of their underlying functions observed in Fig. 3. Despite of not modeling phase variations, the erf and M-METIS phase results in Fig. 4(c) are not completely flat due to the movement of the antenna gain reference point. Finally, it is also noted that the agreement between the evaluated models and PO increases when the specular reflection point lies within the rectangle.

Second, in Scenario B, the carrier frequency is changed to $39 \mathrm{GHz}$ and the $\mathrm{Tx}$ and $\mathrm{Rx}$ antennas from Scenario $\mathrm{A}$ are 


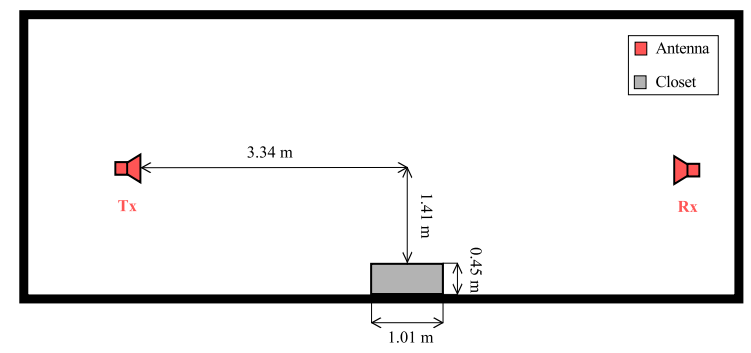

(a)

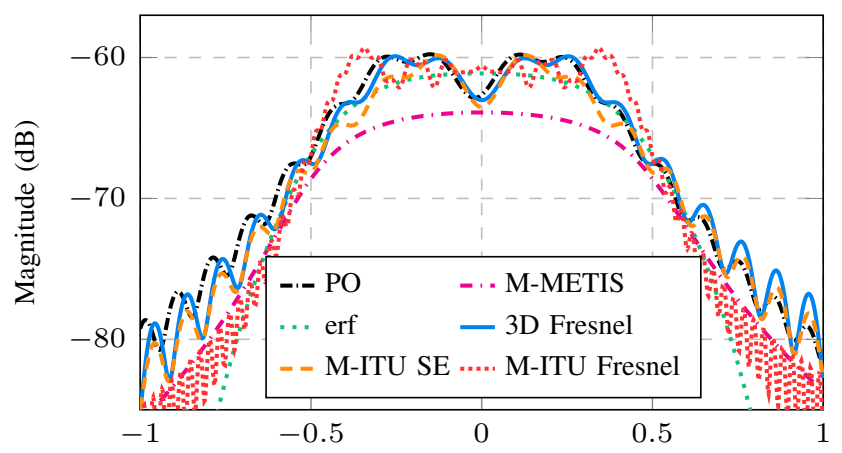

Closet midpoint $\mathrm{x}$-offset from specular reflection point $(\mathrm{m})$

(b)

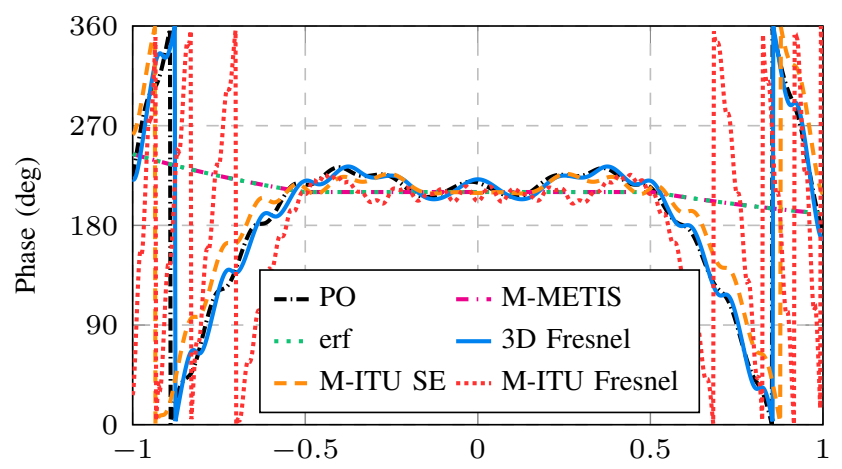

Closet midpoint $\mathrm{x}$-offset from specular reflection point $(\mathrm{m})$

(c)

Fig. 6. Scenario C: moving-along-wall rectangle at $28 \mathrm{GHz}$. (a) Top projection of the zero $\mathrm{x}$-offset situation; (b) magnitude and (c) phase results for different rectangle positions.

placed two meters apart and pointed towards O, see Fig. 5(a), thus producing oblique incidence on the scattering surface. The proposed 3D Fresnel model and the M-ITU Fresnel model now differ in magnitude and, more noticeably, in phase, as can be observed in Figs. 5(b) and 5(c), and more clearly in Table III. Again, the proposed 3D Fresnel model performs best compared to the $\mathrm{PO}$ reference.

Third, Scenario C is based on the $28 \mathrm{GHz}$ Scenario 1 in [33] (reproduced in Fig. 6(a)), originally designed to have the specular reflection point in the midpoint of the surface's width along the line dictated by the antennas' height, and "moves" the surface (modeling the front of a "metallic closet") along a "wall". This provides a test of the performance of the models for linear displacement of the surface around the specular

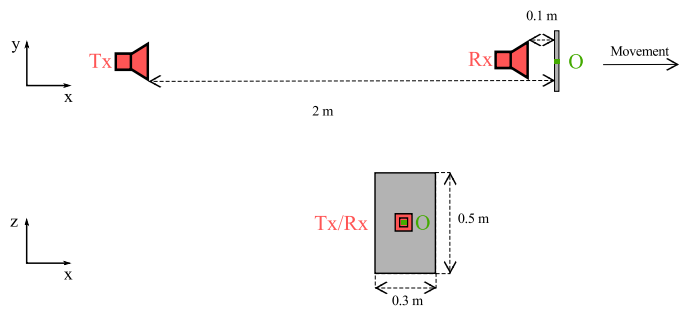

(a)

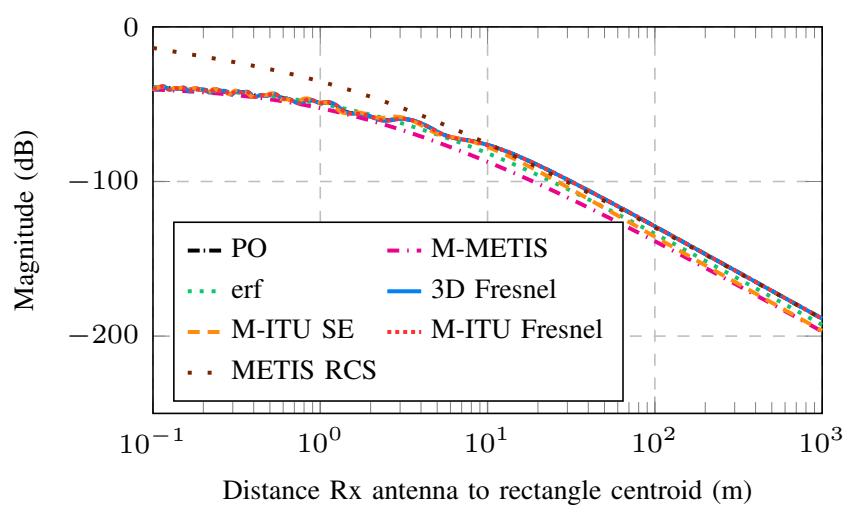

(b)

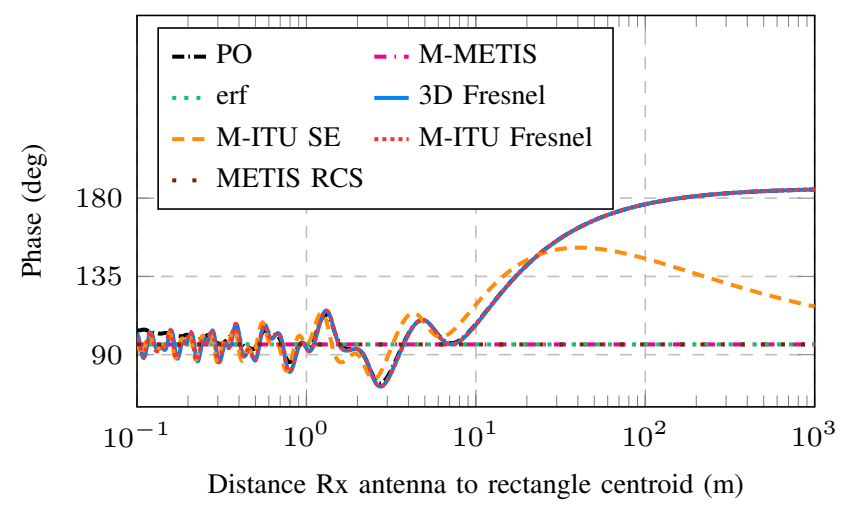

(c)

Fig. 7. Scenario D: moving-away rectangle at $28 \mathrm{GHz}$. (a) (Top) top projection and (bottom) side projection of the starting position of the surface at 0.1-meter distance to the Rx antenna. (b) Magnitude and (c) phase results for different rectangle-to-Rx antenna distance.

reflection point, which gives varying angles of incidence and departure. It can be seen in Fig. 6 and Table III that the MITU Fresnel model deviates considerably from the PO results, whereas the 3D Fresnel proposed model maintains good performance. The disagreement between the M-ITU Fresnel model and the PO reference was expected since the model was defined for small angles between the surface normal vector and the line drawn between Tx and Rx antennas [13, Sec. 5.2.1.2].

Last, Scenario D tests the models for varying distance between the antennas and the scattering surface at $28 \mathrm{GHz}$. In this scenario, see Fig. 7(a), the Tx and Rx antennas are aligned with and pointing towards the rectangle centroid and displaced $2 \mathrm{~m}$ and $0.1 \mathrm{~m}$ away from the origin $\mathrm{O}$, respectively, along the normal of the scattering surface, with the Rx antenna modeled as transparent with respect to radiation from the Tx antenna. 


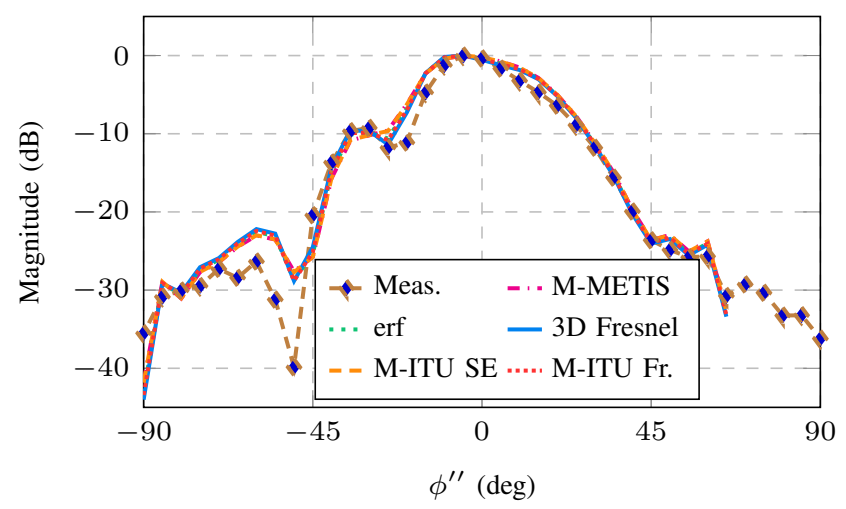

(a)

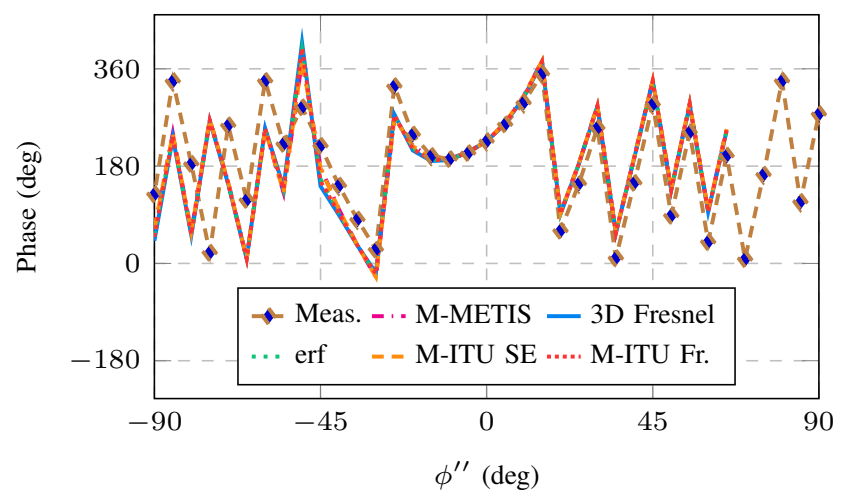

(b)

Fig. 8. Measurement and models results for Scenario 1 in [33]: (a) magnitude and (b) phase results for different Rx antenna pointing directions. In addition, the free-space radiation pattern of the $\mathrm{Rx}$ antenna is shown to facilitate visualization of the objects effects.

The scattered field is calculated for rectangle displacements between 0 and $1 \mathrm{~km}$ from the antenna gain reference point along the the axis formed by the antennas and $\mathrm{O}$. As can be seen in Figs. 7(b) and 7(c), the two Fresnel models appear to perform best, both in the oscillatory Fresnel region and in the far field region, which is confirmed in Table III. It is noted a 90 degree discrepancy between the M-ITU SE model and the Fresnel and PO formulations, in the limit of large distances, due to the consideration of the phase difference between the direct and diffracted waves by the latter approaches, i.e., 90 degrees for spherical waves.

In order to illustrate the limitations of RCS models, the METIS RCS model (with proper compensation for the effective gain of the plate) has been calculated in this scenario. It should be noted that, unlike all other forward-scattering models that were modified following the approach proposed in this paper, the METIS RCS model has been calculated as originally defined by METIS [7, Sec. C.1.5], except for the inclusion of antenna gains. Despite of showing very good magnitude agreement in the far-field, as shown in Figs. 7(b), the METIS RCS model yielded a total WNMSE of 15.2 $\mathrm{dB}$ with respect to the PO reference; an NMSE $38.3 \mathrm{~dB}$ greater than the 3D Fresnel model. This is mainly due to the discrepancy at near-field distances and to the fact METIS RCS is a magnitude-only model. For these reasons, the METIS RCS model has not been calculated in the rest of the near-field

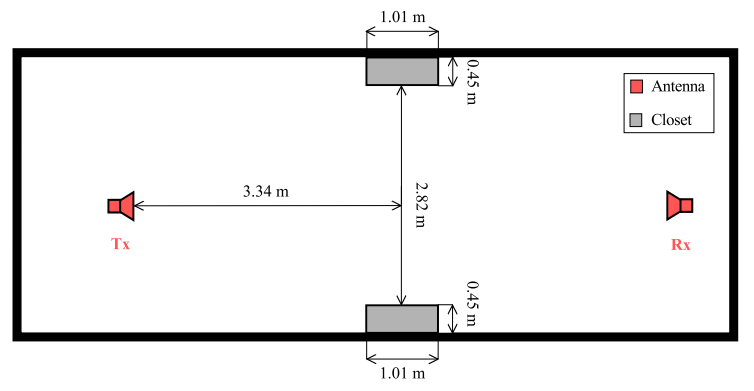

(a)

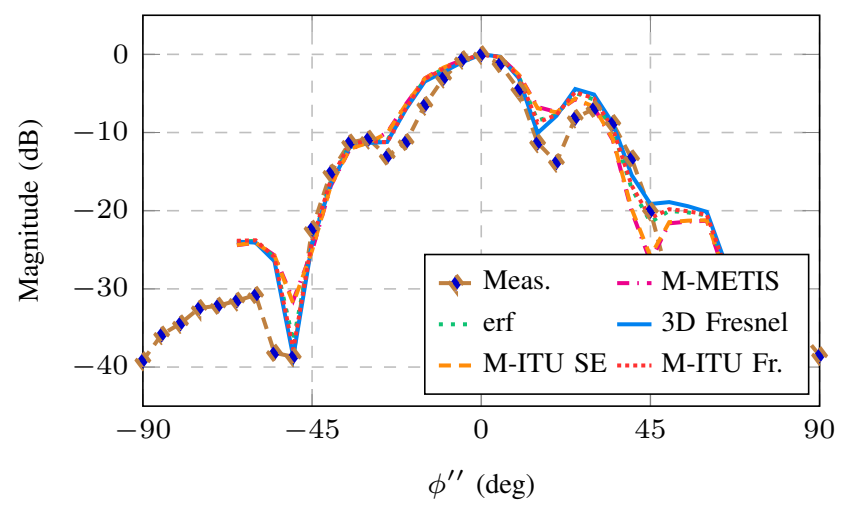

(b)

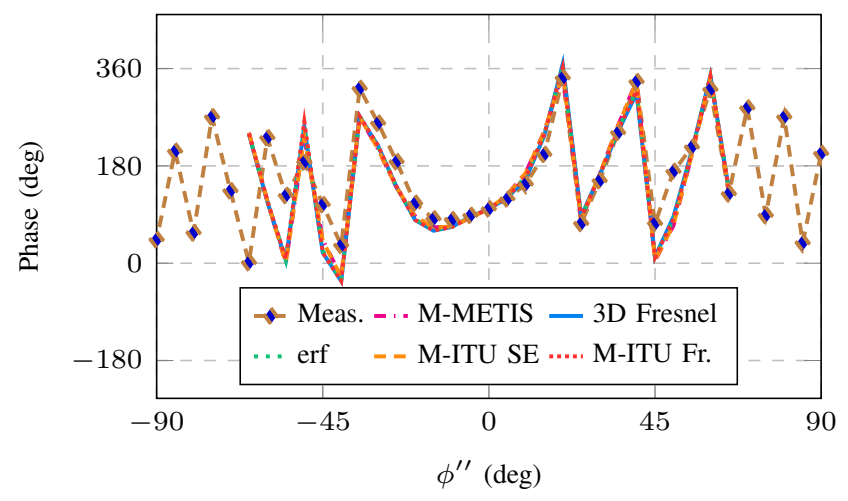

(c)

Fig. 9. Measurement and models results for Scenario 8 in [33]: (a) schematic drawing top projection; (b) magnitude and (c) phase results for different Rx antenna pointing directions. In addition, the free-space radiation pattern of the $\mathrm{Rx}$ antenna is shown to facilitate visualization of the objects effects.

scenarios of this paper (Scenarios A-C).

Finally, as can be observed in Table III, the 3D Fresnel and M-ITU Fresnel models have the best performance in scenarios with normal incidence (Scenarios A and D). However, the 3D Fresnel model maintains the performance for a range of incidence angles whereas M-ITU Fresnel degrades considerably (Scenarios B and C). In addition, the M-ITU SE/MmmMAGIC model also shows good performance regardless of incidence angles. Finally, among the magnitude-only models, the erf model exhibits better performance than the M-METIS model. 
TABLE III

PERFORMANCE COMPARISON OF THE STUDIED MODELS WITH RESPECT TO PO RESULTS

\begin{tabular}{|c|c|c|c|c|c|c|c|}
\hline & & & 3D Fresnel & erf & M-METIS & M-ITU Fresnel & M-ITU SE \\
\hline Scenario & f (GHz) & Interval & NMSE (dB) & NMSE (dB) & NMSE (dB) & NMSE (dB) & NMSE (dB) \\
\hline A & 60 & {$[0,180]$ deg } & -23.3 & -13.3 & -9.9 & -23.6 & -18.2 \\
\hline B & 39 & {$[0,180]$ deg } & -18.3 & -10.6 & -7.3 & -12.4 & -15.2 \\
\hline $\mathrm{C}$ & 28 & {$[-1,1] \mathrm{m}$} & -20.4 & -10.7 & -7.5 & -8.6 & -15.4 \\
\hline Scenario & f (GHz) & Interval & WNMSE (dB) & WNMSE (dB) & WNMSE (dB) & WNMSE (dB) & WNMSE (dB) \\
\hline $\mathrm{D}$ & 28 & {$\left[0.1,10^{3}\right] \mathrm{m}$} & -23.1 & -7.1 & -4.8 & -23.2 & -12.4 \\
\hline
\end{tabular}

\section{Validation Against Measurements}

Here, the proposed models are tested against results from the 28-GHz measurement campaign presented in [33], where configurations consisting of large (in terms of wavelengths) objects were measured in an anechoic chamber. Scenario 1 in [33], reproduced in Fig. 6(a), was designed to have the specular reflection point at the width-dimension midpoint of the $1.01 \times 0.45 \times 1.98$-meter metallic closet. Similar to this, Scenario 8 from [33] introduces a second same-sized closet symmetrically located at the other side of the anechoic chamber, see Fig. 9(a). These two scenarios were selected to test the models on metallic objects providing significant scattering compared to the free space (direct) radiation. Asymmetry in the measured magnitude results is observed in Fig. 9(b) due to Tx antenna misalignment. Hence, the Tx antenna was modelled pointing 3 degrees away from the Rx antenna direction, toward the left closet, as seen from the Tx antenna, i.e, the upper closet in Fig. 9(a), in order to obtain the model results shown in Figs. 8 and 9.

The models results were calculated based on the measured Tx and Rx antenna patterns in [33] and plotted as a function of the Rx antenna pointing direction $\phi^{\prime \prime}$. The results in Figs. 8 and 9 are shown for the angle intervals where measured $\mathrm{Rx}$ antenna pattern data was available for interpolation, given that the original measurements were limited to the $[-90,+90]$ degree interval.

The models show good agreement between measured and calculated results, both in magnitude and phase. All models present similar performance in such scenarios due to two reasons: first, the results are dominated by the LoS contribution, and thus the impact of the difference for the various models is not pronounced and, second, the specular reflection point is contained within the surface.

\section{CONCLUSION}

In this paper, a set of computationally-efficient singlescattering models to account for the backscattering phenomena from finite-size arbitrarily oriented and positioned rectangular surfaces have been presented. The models are fundamentally based on evaluating the phase difference due to the excess path length via the surface edges with respect to minimum path length.

A 3D model based on the use of the Fresnel integrals and a simpler model using the error function have been proposed. Second, the METIS, ITU Fresnel, and ITU
Semi-empirical/mmMAGIC blockage models have been reformulated to calculate backscattered fields. All models can be computed regardless of the position and pointing of the antennas, and position, orientation, size, and electrical properties of the scattering surfaces.

The proposed single-scattering models have been evaluated both against simulations and measurements. All simulations show the 3D Fresnel model to have the best agreement with respect to magnitude and phase compared to a $\mathrm{PO}$ reference model, with the proposed models being orders of magnitude faster than the PO calculations. In addition, good agreement between model and measurements has been shown.

\section{REFERENCES}

[1] J. G. Andrews et al., "What will 5G be?" IEEE J. Sel. Areas Commun., vol. 32, no. 6, pp. 1065-1082, Jun. 2014.

[2] T. S. Rappaport et al., "Millimeter wave mobile communications for 5G cellular: It will work!” IEEE Access, vol. 1, pp. 335-349, 2013.

[3] mmMAGIC, "Measurement campaigns and initial channel models for preferred suitable frequency ranges," mm-Wave based Mobile Radio Access Netw. for 5G Integrated Commun. (mmMAGIC), Tech. Rep. D2.1 v1, Mar. 2016.

[4] C. Oestges et al., "Radio channel modeling for 4G networks," in Pervasive Mobile and Ambient Wireless Communications: COST Action 2100, R. Verdone and A. Zanella, Eds. London: Springer, 2012, ch. 3, pp. 67-147.

[5] D. Solomitckii et al., "Characterizing the impact of diffuse scattering in urban millimeter-wave deployments," IEEE Wireless Commun. Lett., vol. 5, no. 4, pp. 432-435, Aug. 2016.

[6] F. Mani et al., " $26 \mathrm{GHz}$ ray-tracing pathloss prediction in outdoor scenario in presence of vegetation," in Proc. European Conf. Antennas Prop. (EuCAP), Apr. 2018, pp. 1-5.

[7] METIS, "METIS channel models," Mobile and Wireless Commun. Enablers for the 2020 Inf. Soc. (METIS), Tech. Rep. D1.4 v3, Jul. 2015.

[8] M. K. Samimi and T. S. Rappaport, "3-D statistical channel model for millimeter-wave outdoor mobile broadband communications," in Proc. IEEE Int. Conf. Commun. (ICC), Jun. 2015, pp. 2430-2436.

[9] COST IC1004, "White paper on channel measurements and modeling for $5 \mathrm{G}$ networks in the frequency bands above $6 \mathrm{GHz}$," Eur. Cooperation in Sci. and Technol. (COST), Tech. Rep., Apr. 2016.

[10] A. A. Goulianos et al., "Measurements and characterisation of surface scattering at $60 \mathrm{GHz}$," in Proc. IEEE Veh. Technol. Conf. Fall (VTCFall), Sep. 2017, pp. 1-5.

[11] J. Medbo and F. Harrysson, "Channel modeling for the stationary UE scenario," in Proc. European Conf. Antennas Prop. (EuCAP), Apr. 2013, pp. 2811-2815.

[12] mmMAGIC, "Measurement results and final mmMAGIC channel models," mm-Wave based Mobile Radio Access Netw. for 5G Integrated Commun. (mmMAGIC), Tech. Rep. D2.2 v2, May 2017.

[13] ITU-R, "Propagation by diffraction," Int. Telecommun. Union (ITU), Tech. Rep. P.526, Jan. 2018.

[14] J. Kunisch and J. Pamp, "Ultra-wideband double vertical knife-edge model for obstruction of a ray by a person," in Proc. Int. Conf. UltraWideband (ICUWB), vol. 2, Sep. 2008, pp. 17-20. 
[15] Z. Wang, H. Liu, S. Xu, X. Bu, and J. An, "A diffraction measurement model and particle filter tracking method for RSS-based DFL," IEEE J. Sel. Areas Commun., vol. 33, no. 11, pp. 2391-2403, Nov. 2015.

[16] G. R. MacCartney, S. Deng, S. Sun, and T. S. Rappaport, "Millimeterwave human blockage at $73 \mathrm{GHz}$ with a simple double knife-edge diffraction model and extension for directional antennas," in Proc. IEEE Veh. Technol. Conf. Fall (VTC-Fall), Sep. 2016, pp. 1-6.

[17] T. S. Rappaport, G. R. MacCartney, S. Sun, H. Yan, and S. Deng, "Small-scale, local area, and transitional millimeter wave propagation for 5G communications," IEEE Trans. Antennas Propag., vol. 65, no. 12, pp. 6474-6490, Dec. 2017.

[18] H.-J. Li and Y.-W. Kiang, "Radar and inverse scattering," in The Electrical Engineering Handbook, W.-K. Chen, Ed., lst ed., Cambridge, MA: Academic Press, 2005, pp. 671-690.

[19] V. Degli-Esposti, D. Guiducci, A. de'Marsi, P. Azzi, and F. Fuschini, "An advanced field prediction model including diffuse scattering," IEEE Trans. Antennas Propag., vol. 52, no. 7, pp. 1717-1728, Jul. 2004.

[20] F. Fuschini, E. M. Vitucci, M. Barbiroli, G. Falciasecca, and V. DegliEsposti, "Ray tracing propagation modeling for future small-cell and indoor applications: A review of current techniques," Radio Sci. vol. 50, no. 6, pp. 469-485, Jun. 2015.

[21] C. A. Balanis, Advanced Engineering Electromagnetics, 2nd ed. Hoboken, NJ: Wiley, 2012.

[22] ITU-R, "Effects of building materials and structures on radiowave propagation above about $100 \mathrm{MHz}$," Int. Telecommun. Union (ITU), Tech. Rep. P.2040, Jul. 2015.

[23] M. Shafi et al., "5G: A tutorial overview of standards, trials, challenges, deployment, and practice," IEEE J. Sel. Areas Commun., vol. 35, no. 6, pp. 1201-1221, Jun. 2017.

[24] P. Kyösti, J. Lehtomäki, J. Medbo, and M. Latva-aho, "Map-based channel model for evaluation of 5G wireless communication systems," IEEE Trans. Antennas Propag., vol. 65, no. 12, pp. 6491-6504, Dec. 2017.

[25] C. Studer, S. Medjkouh, E. Gönültaş, T. Goldstein, and O. Tirkkonen, "Channel charting: Locating users within the radio environment using channel state information," IEEE Access, vol. 6, pp. 47682-47698, 2018.

[26] E. Björnson, J. Hoydis, and L. Sanguinetti, "Massive MIMO Networks: Spectral, Energy, and Hardware Efficiency," Foundations and Trends Signal Process., vol. 11, no. 3-4, pp. 154-655, 2017.

[27] C. Mollén, E. G. Larsson, U. Gustavsson, T. Eriksson, and R. W. Heath, "Out-of-band radiation from large antenna arrays," IEEE Commun. Mag., vol. 56, no. 4, pp. 196-203, Apr. 2018.

[28] J. Karedal et al., "A geometry-based stochastic MIMO model for vehicle-to-vehicle communications," IEEE Trans. Wireless Commun., vol. 8, no. 7, pp. 3646-3657, Jul. 2009.

[29] D. McNamara, C. Pistorius, and J. Malherbe, Introduction to the Uniform Geometrical Theory of Diffraction, 1st ed. Norwood, MA: Artech House, 1990.

[30] G. D. Durgin, "The practical behavior of various edge-diffraction formulas," IEEE Antennas Propag. Mag., vol. 51, no. 3, pp. 24-35, Jun. 2009.

[31] C. L. Andrews and D. P. Margolis, "Elemental derivation of the Babinet principle in electromagnetic form," Am. J. Phys., vol. 43, no. 8, pp. 672-676, 1975.

[32] D. Parsons, The Mobile Radio Propagation Channel, 2nd ed. Hoboken, NJ: Wiley, 2000.

[33] A. Lahuerta-Lavieja, M. Johansson, G. A. E. Vandenbosch, and U. Gustavsson, "Environment-embedded radiation patterns at millimeterwave frequencies," in Proc. European Conf. Antennas Prop. (EuCAP), Mar. 2019, pp. 1-5.

[34] R. F. Harrington, Field Computation by Moment Methods, 1st ed. Hoboken, NJ: Wiley-IEEE Press, 1993.

[35] S. Liao and R. J. Vernon, "A fast algorithm for computation of electromagnetic wave propagation in half-space," IEEE Trans. Antennas Propag., vol. 57, no. 7, pp. 2068-2075, Jul. 2009.

[36] A. Boag, "A fast physical optics (FPO) algorithm for high frequency scattering," IEEE Trans. Antennas Propag., vol. 52, no. 1, pp. 197-204, Jan. 2004.

[37] "Memoryless nonlinear models," in RF Power Amplifier Behavioral Modeling, D. Schreurs, M. O’Droma, A. A. Goacher, and M. Gadringer, Eds. Cambridge Univ. Press, 2008, ch. 3, pp. 86-135.

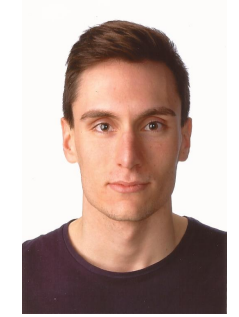

Adrián Lahuerta Lavieja received the M.Sc. degree in telecommunication engineering from the University of Zaragoza, Zaragoza, Spain, in 2016. $\mathrm{He}$ is currently pursuing a Ph.D. degree in electrical engineering at KU Leuven, Leuven, Belgium, within the MSCA H2020 project SILIKA.

Since 2017, he holds a visiting researcher position at Ericsson Research, Ericsson AB, Gothenburg, Sweden, where he is involved in behavioral modeling of radio hardware. His research interest include channel modeling, radio signal processing and behavioral modeling of radio hardware for advanced antenna systems.

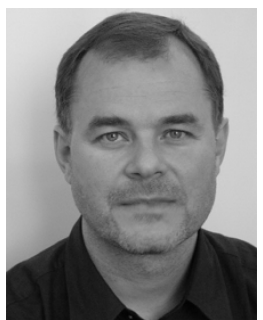

Martin Johansson (M'93-SM'06) received the M.Sc. degree in engineering physics and the Ph.D. degree in electromagnetics from the Chalmers University of Technology in 1986 and 1997, respectively.

He joined Ericsson Research, Ericsson AB, Gothenburg, Sweden, in 1997, where he currently serves as an Expert in antenna technology. His current research interests include antenna technology for mobile communications, antenna system modeling, and deterministic channel modeling.

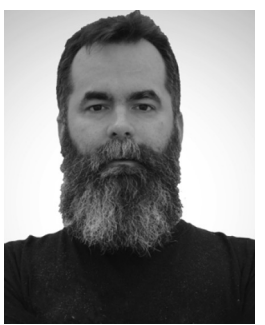

Ulf Gustavsson received the M.Sc. degree in electrical engineering from Orebro University, Örebro, Sweden, in 2006, and the Ph.D. degree from the Chalmers University of Technology, Gothenburg, Sweden, in 2011. His background ranges from power amplifier design to radio signal processing. He is currently a Senior Specialist with Ericsson Research where his research interests include novel radio signal processing techniques for hardware impairment mitigation and behavioral modeling of radio hardware for future advanced antenna systems and communication engineering. Dr. Gustavsson is currently also the lead scientist from Ericsson Research in the Marie Skłodowska-Curie European Industrial Doctorate Innovative Training Network, SILIKA ( http://silika-project.eu/ ).

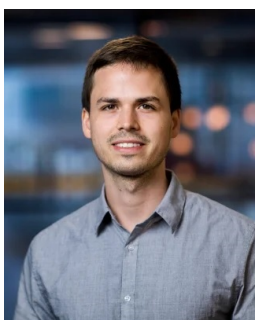

Thomas A. H. Bressner received his B.S. degree in electrical engineering and information technology from the Karlsruhe Institute of Technology (KIT), Germany, in 2012. He obtained in the course of a double master's degree program an M.S. degree in electrical engineering from the Royal Institute of Technology (KTH) in Stockholm, Sweden, in 2015 and an M.S. degree in electronic engineering from Politecnico di Torino (POLITO), Italy, in 2015. Besides the double master's degree, he obtained his third M.S. degree in electrical engineering with a specialization in radio frequencies from Karlsruhe Institute of Technology (KIT), Germany, in 2016. Subsequently, he joined the department of electrical engineering, Eindhoven University of Technology (TUE), as a Ph.D. student and contributes by his work on energy-efficient base stations for mobile communications to the European project SILIKA. 


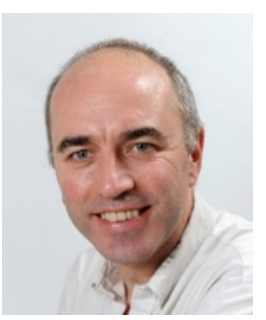

Guy A. E. Vandenbosch (M'92-SM'08-F'13) received the M.S. and Ph.D. degrees in Electrical Engineering from the Katholieke Universiteit Leuven, Leuven, Belgium, in 1985 and 1991, respectively. Since 1993, he has been a Lecturer, and since 2005 a Full Professor at the same university. His research interests are in the area of electromagnetic theory, computational electromagnetics, planar antennas and circuits, nano-electromagnetics, EM radiation, EMC, and bio-electromagnetics. His work has been published in ca. 340 papers in international journals and has led to ca. 390 papers at international conferences. Guy Vandenbosch has been a member of the "Management Committees" of the consecutive
European COST actions on antennas since 1993. Within the ACE Network of Excellence of the EU (2004-2007), he was a member of the Executive Board and coordinated the activity on the creation of a European antenna software platform. After ACE, from 2007 - 2018, he chaired the EuRAAP Working Group on Software. In the period 1999-2004, he was vice-chairman, in the period 2005-2009 secretary, and in the period 2010-2017 chairman of the IEEE Benelux Chapter on Antennas en Propagation. In the period 2002-2004 he was secretary of the IEEE Benelux Chapter on EMC. In the period 2012-2014, he was secretary of the Belgian National Committee for Radio-electricity (URSI), where he is also in charge of commission E. Guy Vandenbosch is a fellow of the IEEE. From September to December 2014, he was a visiting professor at Tsinghua University, Beijing, China. Since 2017 he is a member of the IEEE Electromagnetics Award Committee. 